\title{
Healthy eating: an NHS priority $A$ sure way to improve health outcomes for NHS staff and the public
}

\author{
Aseem Malhotra, ${ }^{1,2}$ Mahiben Maruthappu, ${ }^{3}$ Terence Stephenson ${ }^{4}$
}

Tackling the obesity epidemic and its associated adverse health consequences is one of today's important public health challenges. Obesity directly costs the National Health Service (NHS) about $£ 6$ billion per year. Direct and indirect costs of diabetes are estimated to be $£ 24$ billion and are likely to double over the next 20 years. ${ }^{1}$ Some fundamental misunderstandings among the medical and healthcare community and lay public inhibit the implementation of effective interventions. Our decisions about the food we buy and what we eat are often automatic and made without full conscious awareness. ${ }^{2}$ For example, despite wanting to lose weight, we're still tempted to buy the brightly packaged chocolate bar at the checkout till.

The lifestyle hierarchy places diet as a powerful common determinant of cardiovascular disease, obesity, type 2 diabetes and several cancers. ${ }^{3}$ The Lancet Global Burden of Disease Study reports that poor diet contributes to more disease than physical inactivity, smoking and alcohol combined. ${ }^{4}$ Recent scientific advances support a number of specific dietary targets to be prioritised for the prevention of cardiovascular disease. Several are aimed at increasing consumption of healthy foods, with a number to reduce the consumption of harmful ones. The $\alpha$-linoleic acid, polyphenols and omega-3 fatty acids found in abundance in nuts, fruit, vegetables, olive oil and oily fish rapidly exert positive health effects by attenuating thrombosis and inflammation, ${ }^{5}$ and it is estimated that increasing population consumption of fruit and vegetables by one portion per day and nut consumption by two servings a week would prevent 5.2 million deaths from cardiovascular disease globally within just 1 year. $^{6}$ Conversely, the consumption of trans-fats commonly found in

${ }^{1}$ Department of Cardiology, Frimley Park Hospital, Surrey, UK: ${ }^{2}$ Academy of Medical Royal Colleges, London, UK; ${ }^{3} \mathrm{NHS}$ England, London, UK; ${ }^{4}$ Institute of Child Health, UK Academy of Medical Royal Colleges, London, UK

Correspondence to Dr Aseem Malhotra, Academy of Medical Royal Colleges, 10 Dallington Street, London, EC1V 0DB; aseem_malhotra@hotmail.com fast food can rapidly increase C-reactive protein and other inflammatory markers within weeks. ${ }^{7}$ Reducing consumption of sugary drinks by $15 \%$ would prevent 180000 people from becoming obese in the UK in the same time period and save $£ 275$ million for re-investment in the NHS. $^{8}$

Real progress towards combating the obesity epidemic and diseases associated with diet will only start once a root cause - the need for a healthier food environment-is understood. The success of the junk food industry depends on the availability of their products to anyone, anywhere, at any time. Tackling the obesity burden requires collective action, and there is no single silver bullet, but there is an established hierarchy of effectiveness. The Centres of Disease Control health impact pyramid shows that, by changing the context-that is, the food environment - so that individuals' choices about what to eat default to healthy options will have a far greater impact on population health than counselling or education. Healthy choice must become the easy choice. ${ }^{9}$ Changing the context of smoking by insisting on smoke-free buildings has had a huge impact on reducing smoking prevalence and improving health. Within just 1 year of the introduction of smoke-free legislation in Scotland, there was a $17 \%$ reduction in admissions for heart attacks and a $6 \%$ decrease in out-of-hospital cardiac deaths. ${ }^{10}$

The Boorman Report demonstrated that better staff health and well-being is associated with better organisational performance and improved patient outcomes. ${ }^{11}$ The NHS is the primary interface with 'health' for most of the population, and so the workforce environment and the attitudes to health and well-being and diet of NHS staff can impact on health promotion. However, the oversupply of cheap, high-energy but nutrient-poor food and drink even pervades the institutions that should be promoting positive health messages-our hospitals. Confectionary, crisps and sugary drinks are available to staff and patients through vending machines in hospital corridors and to bed-bound patients via hospital trolleys. Also, many hospitals have high-street fast-food franchises on site. Thus acceptability and consumption of such foods is legitimised by being in a healthcare setting. Moreover, reduced availability of healthy food options limits choice for many NHS staff, half of whom are estimated to be overweight or obese. ${ }^{12}$ Educating people about healthy eating is difficult when the food environment is so unhealthy.

Strategies that prevent excessive weight gain for children and adults by reducing consumption of unhealthy foods should be welcomed. However, focusing only on weight loss by any means is a missed opportunity and so potentially harmful. Health practitioners should move beyond the traditional, well-intentioned but limited dietary advice-to 'eat less and move more'-and be more specific and evidence based. Regular physical activity consisting of a 30 min brisk walk per day has tremendous cardiovascular, bone and musculoskeletal benefits, but the link to obesity is weak ${ }^{13}$ and its benefits are undermined by poor dietary habits. A recent longitudinal cohort study involving 175 countries showed that, for every additional 150 sugar-based kilocalories consumed daily (typical of a can of cola) compared with calories from another source, there was an 11-fold increase in the prevalence of developing type 2 diabetes independent of body mass index and physical activity levels. ${ }^{14}$ You can't outrun a bad diet.

Rapid weight loss and regain is detrimental to health. Such 'weight cycling' contributes to hypertension, insulin resistance and dyslipidaemia resulting in increased mortality risk and worse cardiovascular outcomes. The weight loss industry, which promotes calorie restriction rather than good nutrition, generates US $\$ 58$ billion in revenue annually in the USA even though long-term follow-up studies (up to 5 years) show that most people on rapid diets regain much of the lost weight; about two-thirds do not achieve health benefits and put on weight whether or not they maintain their diet or exercise programme. ${ }^{15}$ In contrast, with advice on a low-fat diet, the PREDIMED primary prevention randomised controlled trial, which included 7500 high-risk individuals initially free of cardiovascular disease, found that an energy-unrestricted Mediterranean diet $(41 \%$ total fat) supplemented with extra-virgin olive oil or nuts achieved a $30 \%$ reduction in major cardiovascular events, with event reduction occurring even within 3 months. ${ }^{16}$ A key finding from analysis of PREDIMED subgroups showed that dietary intervention achieved 
consistently large reductions in cardiovascular disease risk irrespective of weight.

Concentrating on good nutrition rather than counting calories will not only improve metabolic health in the short term but may promote sustained weight loss. A recent randomised study published in the Annals of Internal Medicine showed that an energy-unrestricted higher-fat and low-refined-carbohydrate diet (restricting carbohydrates without fibre) resulted in more weight loss and a better lipid profile than a low-fat diet at 1 year. ${ }^{17}$

Introducing evidence-based nutrition into postgraduate training programmes and undergraduate curriculums would increase doctors' and nurses' understanding of the science of healthy eating and allow a much more informed dialogue between doctors, nurses and their patients. Good nutritional advice is particularly important for those at special risk-for example, those with diabetes. Tight glycaemic control reduces the risk of microvascular complications, but its impact on macrovascular complications of heart attack and stroke is negligible. And so people with diabetes told that they can eat what they like as long as they take their medication may be unnecessarily missing an important opportunity to keep healthy. Adopting a Mediterranean diet after a myocardial infarction is almost three times as effective at reducing mortality than taking a statin. ${ }^{18}$ But how many people are actively given this information, which might encourage them to improve their diet while also adhering to evidence-based pharmacotherapy?

Although gradual weight reduction and physical activity have merits, dietary improvement is a much more effective approach to health improvement in both the short and long term. The NHS as an employer is in a key position to set a national example, to support 1.4 million staff to stay healthy, and serve as 'health ambassadors' in their local communities. While three-quarters of NHS trusts say they offer support to help staff to quit smoking, only about a third offer them support in keeping to a healthy weight and diet. Three-quarters of hospitals do not offer healthy food to staff working night shifts. $^{19}$

Informing patients and the public how diet can improve health is the responsibility of all healthcare workers, who should lead by example. To do this, health workers need to have education, training and access to information.
Box 1 Proposal for actions for

healthy eating in the NHS

- Make information about the evidence base for healthy diets easily available to NHS staff and patients

- NHS employers to implement the Workplace Wellbeing Charter ${ }^{21}$ and require commissioners to consider this when assessing tenders

- Implement National Institute of Health and Care Excellence guidance on promoting healthy workplaces throughout the NHS

- Reduce access to processed foods high in fat, salt and sugar on NHS premises

- Provide healthy options for all staff, including night staff

- Call for NHS institutions to objectively monitor and publish sales and quantities of foods deemed unhealthy, in addition to the degree of adherence to national food standards

The recently published NHS Five Year Forward View commits the NHS to a number of initiatives.

It is time to put the evidence base that dietary changes can rapidly and substantially improve health outcomes into the heart of the NHS (box 1). ${ }^{20}$ Offering NHS staff the opportunity to make their diets healthier would be a good start to improve the health of the NHS workforce and their patients. This model could then be adopted into other workplace environments.

Contributors AM wrote the initial draft, with further addition/editing by MM and TS.

Competing interests None.

Provenance and peer review Commissioned; internally peer reviewed.
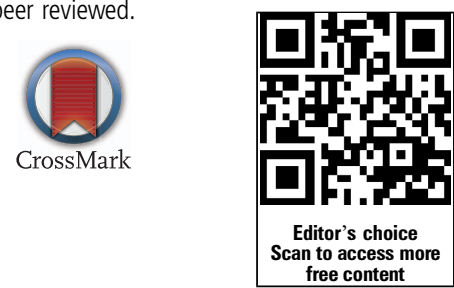

To cite Malhotra A, Maruthappu M, Stephenson T. Postgrad Med J 2014;90:671-672.

Received 22 October 2014

Accepted 30 October 2014

Published Online First 17 November 2014

Postgrad Med J 2014;90:671-672.

doi:10.1136/postgradmedj-2014-133103

\section{REFERENCES}

1 Hex N, Bartlett C, Wright D, et al. Estimating the current and future costs of Type 1 and Type 2 diabetes in the United Kingdom, including direct health costs and indirect societal and productivity costs. Diabetic Medicine 2012:29:855-62.

2 Marteau TM, Ogilvie D, Roland M, et al. Judging nudging: can nudging improve population health? BMJ 2011;342:d228.

3 United Nations. Draft outcome document of the high-level meeting on the prevention and control of non-communicable diseases. 2011. http://www. ncdalliance.org/sites/default/files/resource_files/UN\% 20High-Level\%20Summit\%20Zero\%20Draft.pdf

4 Global Burden of Diseases, Injuries, and Risk Factors Study 2013. The Lancet, 22 Jul 2014. http://www. thelancet.com/themed/global-burden-of-disease

5 Chakrabarti S, Freedman JE. Review: Nutriceuticals as antithrombotic agents. Cardiovasc Ther 2010;28:227-35.

6 Mozaffarian D, Capewell S. United Nations' dietary policies to prevent cardiovascular disease. BMJ 2011;343:d5747

7 Wallace S, Mozaffarian D. Trans-fatty acids and non lipid risk factors. Curr Atheroscler Rep 2009;11:423.

8 Briggs ADM, Mytton OT, Kehlbacher A, et al. Overall and income specific effect on prevalence of overweight and obesity of $20 \%$ sugar sweetened drink tax in UK: econometric and comparative risk assessment modelling study. BMJ 2013;347: f6189.

9 Frieden TR. A framework for public health action: the health impact pyramid. Am J Public Health 2010;100:590-5.

10 Meyers DG, Neuberger JS, He J. Cardiovascular effect of bans on smoking in public places: a systematic review and meta-analysis. J Am Coll Cardiol 2009;54:1249-55.

11 NHS Health and Well-being - Final Report. Nov 2009. http://www.nhshealthandwellbeing.org/pdfs/ NHS\%20Staff\%20H\&WB\%20Review\%20Final\% 20Report\%20VFinal\%2020-11-09.pdf

12 Royal College of Physicians. Action on obesity: comprehensive care for all. Jan 2013. http://www. rcplondon.ac.uk/sites/default/files/action-on-obesity. pdf

13 Swinburn B. Increased energy intake alone virtually explains all the increase in body weight in the United States from the 1970s to the 2000s. (Abstract T1: RS3.3.) European Congress on Obesity; 6-9 May 2009; Amsterdam.

14 Basu S, Yoffe P, Hills N, et al. The relationship of sugar to population-level diabetes prevalence: an econometric analysis of repeated cross-sectional data. PLOS ONE 2013:8:e57873.

15 Bacon L, Aphramor L. Weight science: evaluating the evidence for a paradigm shift. Nutr J 2011:10:9.

16 Estruch R, Ros E, Salas-Salvadó J, et al. Primary prevention of cardiovascular disease with a mediterranean diet. $N$ Engl J Med 2013:368:1279-90.

17 Bazzano LA, Hu T, Reynolds K, et al. Effects of low-carbohydrate and low-fat diets: a randomized trial. Ann Intern Med 2014;161:309-18.

18 Quaas J. Mediterranean diet for secondary prevention after heart attack. 17 Sep 2010. http://www.thennt. $\mathrm{com} / \mathrm{nnt} /$ mediterranean-diet-for-post-heart-attack-care

19 Get serious about obesity or bankrupt the NHS Simon Stevens. NHS England, 17 Sept 2014. http:/ www.england.nhs.uk/2014/09/17/serious-aboutobesity

20 NHS England, Public Health England, Health Education England, Monitor, Care Quality Commission, NHS Trust Development Authority. Five Year Forward View. 2014

21 Health@work - The Charter. http://www. healthatworkcentre.org.uk/The-Charter-i3.html 